\title{
Simulation Design and Performance Analysis for VolP in Cognitive Radio Networks
}

\author{
Tamal Chakraborty \\ Dept. of Electronics and \\ Telecommunication \\ Engineering \\ Jadavpur University, \\ Kolkata-700032, India
}

\author{
Iti Saha Misra \\ Dept. of Electronics and \\ Telecommunication \\ Engineering \\ Jadavpur University, \\ Kolkata-700032, India
}

\author{
Salil Kumar Sanyal \\ Dept. of Electronics and \\ Telecommunication \\ Engineering \\ Jadavpur University, \\ Kolkata-700032, India
}

\begin{abstract}
Increasing popularity of VoIP systems has witnessed applications in emerging technologies like Cognitive Radio Networks (CRN). The stringent QoS requirements in VoIP coupled with complexities in CRN have initiated intensive research in the field of performance analysis and optimizations guided by simulation results. However, in the absence of any standard model of VoIP over CRN, accuracy and credibility of the simulation output are strongly dependent on proper design of the simulation model that must have a strong mathematical foundation. The objective of this paper is to build standard models for VoIP in CRN and successfully implement VoIP applications over CRN domain, which will serve as initial point for development with respect to all future simulation studies in VoIP over CRN category. Initially, models of VoIP in CRN are developed using OPNET Modeler 16.0.A following distributed architecture in single-channel and multi-channel scenarios and further in Visual C++ adhering to the principles of centralized architecture. The models are validated by comparison of simulation results obtained in both platforms. The underlying mathematical model behind the design is established and the critical factors pertaining to both VoIP and CRN domain are extensively analyzed.
\end{abstract}

\section{General Terms}

Wireless Communication.

\section{Keywords}

Voice Over IP, Cognitive Radio Network, Simulation, OPNET Modeler 16.0.A., Visual C++.

\section{INTRODUCTION}

Wireless communication has evolved with time thereby witnessing consistently increasing number of users and higher bandwidth requirement of data and multimedia technologies that has adversely affected the availability of frequency spectrum. Cognitive radio [1] addresses this problem of spectral congestion by introducing opportunistic usage of frequency bands that are not heavily occupied by licensed users [1-3]. Considering the popularity of VoIP owing to low maintenance and operating costs [4], increased support for more users while using limited radio resources [5] is of primary concern. Therefore, VoIP implementation over cognitive radio network (CRN) has bright prospect that must be explored thoroughly. However, real-time constraints imposed by delay sensitive communication add to the complexities of CRN design and must be carefully addressed.

Although considerable research studies have ventured into the challenges of CRN, analysis and optimization of VoIP calls in
CRN have witnessed relatively few works. VoIP capacity analysis, for example, has been done over a cognitive radio model in [5] and [6] through a queuing model based on the MMPP traffic flow and a Markov channel model without any retransmission. This work has been extended over single and multiple channels in [7]. Delay analysis for real-time traffic has been performed with respect to cognitive radio sensor networks in [8]. The potential contributions of cognitive radio to spectrum pooling are highlighted in [9] which outline an initial framework for formal radio-etiquette protocols for flexible mobile multimedia communications. Further, adaptive packet scheduling algorithm based on priority based queues for Quality of Service (QoS) maintenance in real-time traffic has been proposed in [10].

In absence of any standard for VoIP in CRN implementation, such research is based on simulation studies and mathematical analysis. However, the model description of VoIP over CRN in all such works lacks transparency and clarity and also requires proper documentation followed by verification and validation. It has been observed that simulation in telecommunication and networking often lacks credibility due to lack of appropriate use of pseudo-random generators and in-depth analysis of simulation output data [11]. Therefore, design of proper simulation model is as crucial as the analysis and optimization of challenges involved in VoIP over CRN domain. Moreover, the simulation output from the designed model must be analyzed using mathematical tools and validated accordingly to be utilized for future research globally [12].

This paper aims to develop models of VoIP in CRN in different simulation platforms to help researchers with further exploration in this realm. Keeping pace with rapid and continuous development in this domain, several modifications must be made in the proposed models to apply novel, adaptive and cross-layer strategies. Moreover, mathematical models should be modified accordingly in every network layer to support advanced opportunistic mode of communication. Performance in different types of networks can be further studied by placing such customized nodes in already established network models.

The problem is defined in Section 2. A model for VoIP over CRN is designed for both single and multiple channel scenarios following distributed architecture in Section 3. Section 4 provides another implementation of a model of VoIP in CRN by adhering to principles of centralized architecture. Advantages of proposed models along with areas of further improvement are discussed in respective sections. Validation of model design is performed in Section 5 by comparison of simulation output from developed models. The 
underlying mathematical model behind the design is established in Section 6. Extensive analysis of the critical attributes in the developed models is carried out in Section 7. Finally, the work is summarized in Section 8.

\section{PROBLEM DEFINITION}

The objective of this paper is to develop efficient models of VoIP over CRN in different simulation platforms that will help in performing analytical studies and implementing proposed optimizations by suitable modifications to the proposed models. Adhering to the principles of Design Science methodology [13], the primary aim of this work is analysis of problem domain followed by development and evaluation of simulation models to study behavior of real-time VoIP transmission in CRN.

The driving factor behind implementation of VoIP in CRN is that link utilization and hence, capacity of VoIP system must be increased along with enhancement in call quality [6]. Let $L$ be link utilization with respect to time and $M$ be derived expression from Mean Opinion Score (MOS). Let $f(L, M)$ be the function of $L$ and $M$. Therefore, the objective is to maximize $f(L, M)$. Hence, the objective function can be mathematically denoted by (1)

$$
T=\max [f(L, M)]
$$

Active networks like VoIP transmission in CRN embed computational capabilities into conventional networks, thereby massively increasing the complexity. Therefore, simulation has scored over traditional analytical methods in analyzing active networks [14]. This necessitates appropriate design of models in simulation platforms. The basic principle behind proposed simulation model is shown in Figure 1. The primary user (PU) generates traffic at uniform distribution interval between $5 \mathrm{sec}$ to $10 \mathrm{sec}$. The secondary user (SU) is VoIP user and implements G.711 codec [15]. The PU is licensed user with priority to use the channel. But it does not always occupy channels and SUs as cognitive radio users are permitted to use these channels in absence of PU. SUs sense channels in sensing period and start transmission in transmission period only when PU is inactive.

\section{DESIGN OF SIMULATION MODEL IN OPNET MODELER 16.0.A}

Initially, a simple VoIP over CRN model is designed in OPNET Modeler 16.0.A [16] following distributed architecture [17]. OPNET Modeler 16.0.A is chosen as the simulation platform as it offers highly customizable nodes along with options ranging from traffic distribution and network parameters to cross-layer architecture based operational modes and collection of wide range of statistical results.

\subsection{Design in a Single-Channel Scenario}

Initially, a model for VoIP in CRN is designed in OPNET Modeler 16.0.A with respect to a single channel scenario following the principles of distributed architecture.

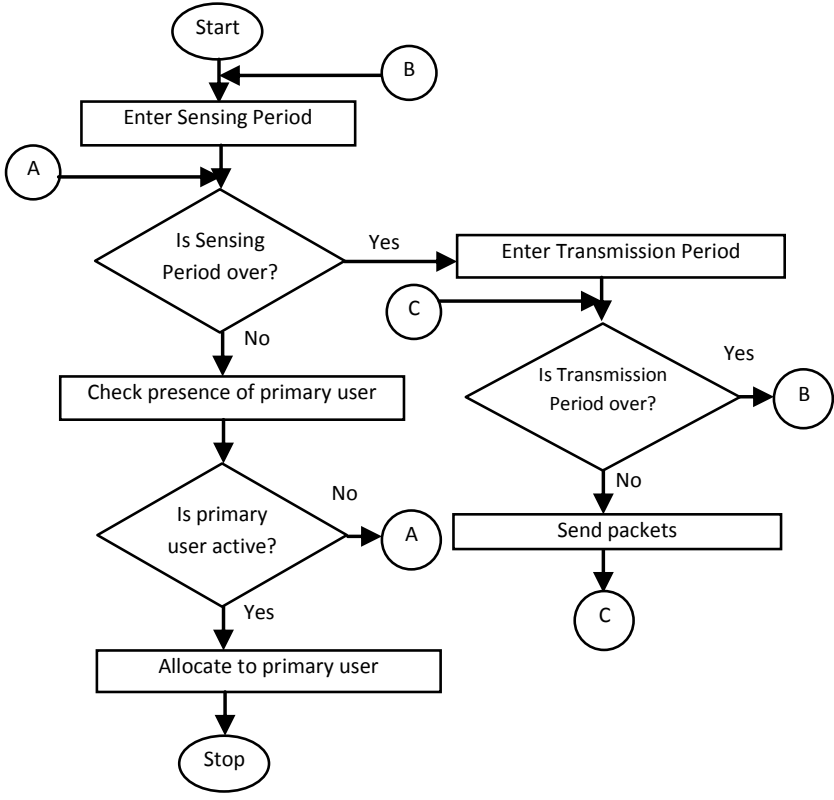

Figure 1: Flowchart depicting the proposed approach

\subsubsection{Overview of the Model}

The node model for SU as designed in OPNET Modeler 16.0.A is shown in Figure 2 (a). The application layer node is the VoIP node responsible for managing VoIP transmissions. This node is followed by Real-time Transport Protocol (RTP), User Datagram Protocol (UDP) and Internet Protocol (IP) nodes. The functionalities of network layers are incorporated in process models corresponding to each node in node model. VoIP_sink node acts as sink for packets already received and processed accordingly. The MAC_Controller node acts as link layer node and cooperates with physical layer node which is involved in sensing, transmission and reception. Spectrum management functionalities like spectrum sensing and spectrum handoff must work in collaboration with the communication protocols [17] and hence such cross-layer architecture is implemented in this paper.

The process model corresponding to MAC_Controller node is shown in Figure 2 (a). It consists of sense and transmit processes that respectively sense and transmit packets according to the principle stated above. Single radio architecture is implemented in sensing principle, where a specific time slot is allocated for spectrum sensing. Thus, only certain accuracy can be guaranteed for spectrum sensing results. It also results in decrease in spectrum efficiency as some portion of available time slot is used for sensing instead of data transmission [18]. The advantage of single radio architecture is its simplicity and lower cost [2], both of which are essential for low cost VoIP communication. Moreover, modeling of sensing is based on energy detection based radiometry [19] or periodogram. The advantages include low computational and implementation complexities that make the model simpler with less algorithmic delays. It is also generic as receivers need no knowledge on PU's signal [2]. However, it may lead to false alarms and precautions must be taken. Further, VoIP packets are designed following protocol formats in each layer. 


\subsubsection{Discussion of Simulation Results}

The principle of maximum throughput for secondary (VoIP) users with negligible interference to the PUs is followed in this model. Accordingly, sensing and transmission time for SUs are varied.

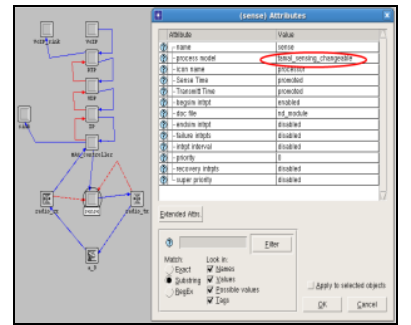

(a)

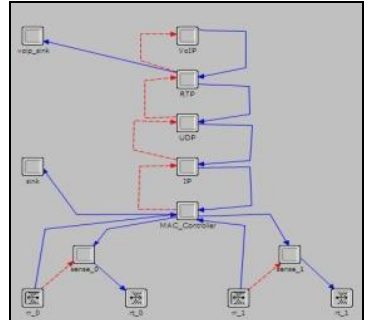

(b)
Figure 2. Node Model of VoIP over CRN in OPNET Modeler 16.0.A for (a) single (b) multi channel scenario

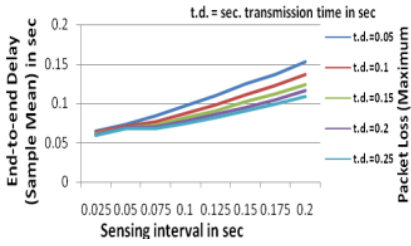

(a)

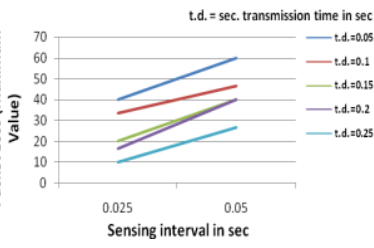

(b)
Figure 3. Variation in (a) end-to-end delay (sample mean), and (b) packet loss (maximum value) with sensing and transmission intervals

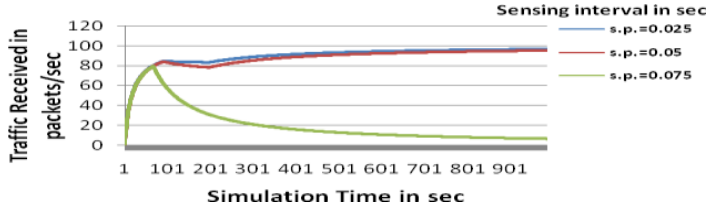

Figure 4. Variation in traffic received for varying sensing intervals

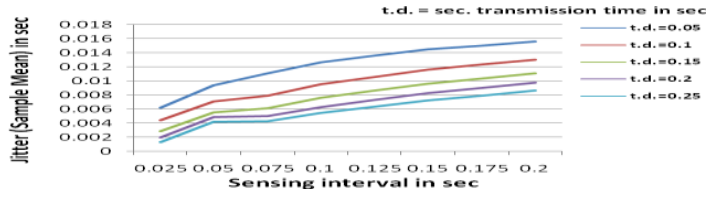

Figure 5. Variation in jitter (sample mean) with sensing and transmission intervals

As observed from Figure 3 (a), mean end-to-end delay for VoIP calls in SUs increases with increase in sensing period due to increase in waiting time for packets waiting to be transmitted. This delay is decreased with increase in secondary transmission period, thereby creating a favorable environment for VoIP transmission. It is further witnessed from Figure 3 (b) that increase in sensing period increases SU packet loss which increases with decrease in secondary transmission period. Throughput degradation for SUs is recorded in Figure 4 with increase in sensing period. A sharp decline in received total traffic with increase in sensing interval denotes that there must be a maximum bound on the sensing interval for successful VoIP transmission. Finally, it is observed from Figure 5 that jitter increases with increase in sensing period and decrease in secondary transmission period.
Thus study of simulation results makes it clear that sensing and transmission intervals have profound effect on QoS of VoIP. It is evident that sensing time must have upper and lower bounds to allow successful VoIP communication without PU interference Solution to this problem includes increasing sensing period resulting in low SU throughput. Short sensing and transmission cycle is another solution that increases jitter in voice traffic.

\subsection{Design in a Multiple-Channel Scenario}

The work is extended in multiple-channel scenario with the assumption that at any point of time, atleast one channel is free for transmission for certain time duration. However, suitable modifications must be made to this model to include effects of various MAC protocols in the domain of free channel allocation.

\subsubsection{Model Overview}

The modified node model for $\mathrm{SU}$ as designed in OPNET Modeler 16.0.A is shown in Figure 2 (b). The node model as described in Section 3.1.1 is updated to include another set of transmitter and receiver that operates in a different channel. This necessitates design of two sense nodes for two channels. Therefore, MAC_Controller node is developed accordingly to switch to the free channel when the other channel is busy. The other nodes along with packet formats are kept similar to the ones described in Section 3.1.1. Single-radio architecture and radiometry as discussed in Section 3.1.1 are applied to this model.

\subsubsection{Study of Simulation Output}

The developed model is used to analyze VoIP performance in CRN following the principle as stated in Section 2. The channel throughput is shown in Figure 6 (a) for each channel from the sender end. Sense and transmit durations are kept at $1 \mathrm{sec}$. It is observed that in presence of PU, channel 2 initiates VoIP transmission and hence simultaneous transmission is observed in both channels.

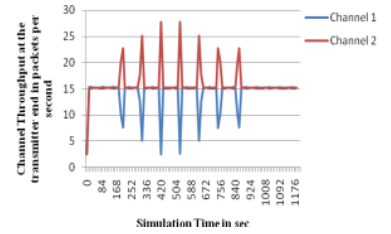

(a)

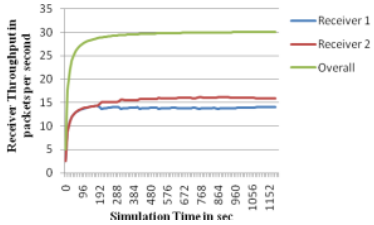

(b)
Figure 6. Variation in (a) channel throughput at the transmitter, and (b) throughput (time average) at the receiver for 2 channels in CRN in ongoing VoIP session

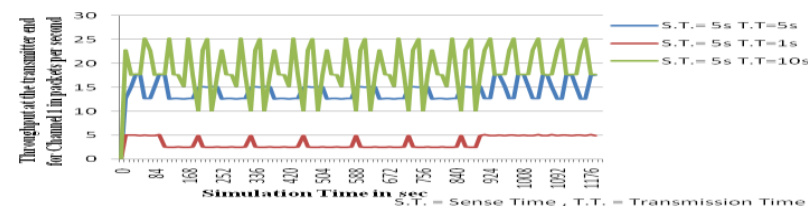

Figure 7. Variation in channel throughput at the transmitter end for channel 1 with sensing and transmission intervals in CRN for an ongoing VoIP session 


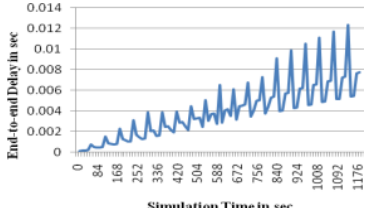

(a)

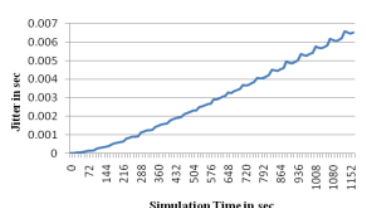

(b)
Figure 8. Variation in (a) end-to-end delay, and (b) jitter for an ongoing VoIP session

Figure 6 (b) records time average view of channel throughput at the receiver end. It is noted that throughput from channel 1 decreases with rise in throughput in channel 2 with time due to high PU activity. However, multi-channel setup ensures successful packet reception from both channels as indicated from overall throughput of the receiver. For different sensing and transmission intervals, it is observed from Figure 7 that channel throughput increases with increasing transmission duration. It is clearly observed in Figure 8 (a) and Figure 8(b) that end-to-end delay and jitter increase with time respectively. This is because number of occurrences with respect to channel switching increases with increased PU activity and hence both delay and jitter increase.

\subsection{Benefits of the Designed Models}

The generic mechanism for development and subsequent maintenance of simulation model requires software engineering concepts along with application expertise. Hence, Prototype model [20] has been implemented in our workflow as it provides us with crude system model that serves as focal point of analysis.

Moreover, multi-paradigm modeling [21] is followed while designing models in OPNET Modeler 16.0.A. The system is considered to be a collection of objects with processes initiating state changes. The advantage is that parallel processing of the object behavior is ensured that is mandatory in simulations involving long runs [11]. However, OPNET Modeler 16.0.A supports conservative approach of system modeling [22] with potential for deadlock and hence care must be taken to prevent it.

The developed simulation models can be used for analysis of the existing algorithms in the field of VoIP performance in $\mathrm{CRN}$, experimentation and application of new algorithms with appropriate modifications to the developed model and as training tools to acquaint researchers with ideas of VoIP implementation in CRN. The simulation output can be analyzed quantitatively and qualitatively along with post simulation animation [23] that visualizes input, internal and output behavior of designed models.

\section{DESIGN OF SIMULATION MODEL IN VISUAL C++}

The work is extended in the domain of model design for VoIP over CRN in a single channel scenario following the centralized architecture. Visual C++ [24] has been chosen for this work as it is the foremost language used in simulation today [25].

\subsection{Overview of the Model}

Both PUs and SUs are modeled based on the fundamental principle of cognitive radio cycle as described in Section 2. The basic modules of developed model are described as follows.
- long int* genNumber (long int time): This module determines the primary traffic distribution. It generates the timestamps that mark the onset of primary traffic arrival.

- int genPacket (int time): This module calculates the primary transmission time. Depending on applications and traffic distributions, the output will vary.

- void allocSec (int time): This module allocates transmission time for SU. It is assumed that there are SUs available for transmission to maximize channel utilization [17].

- int checkPri (long int $j$, long int t_time, long int *flag_random, long int *num_random): This module is the most significant module and is involved in sensing of PU arrival and taking appropriate action. It has submodules that sense the presence of PU and allocate time slots accordingly.

- void showMetrics (): This module displays the metrics of both CRN and VoIP domain. The most significant parameters include total sense time and primary and secondary transmission time elapsed, total number of packets (primary and secondary) at buffer and lost packets, throughput, etc.

- void main(): It serves as initial and final point of execution of VoIP over CRN model. It calls appropriate modules, maintains status of time slot and calculates the metrics.

This model is based on centralized client-server based design. Cross-layer architecture is again followed in this model along with single radio architecture as described in Section 3.1.1.

\subsection{Study of Simulation Data}

Simulation is carried out in the model as described in Section 4.1 for analysis of VoIP deployment over CRN. It is observed from Figure 9 (a) that increase in sensing interval and decrease in secondary transmission interval increase the number of outstanding packets for SUs in buffers. Therefore, sensing period must have a maximum limit to avoid packet loss due to buffer overflow. However, delay increases degrading VoIP call quality.

The scenario varies from PU perspective. For low PU activity, it is noticed that number of sensing intervals where PU is absent or goes undetected is more. These are wasted time intervals for SUs as they abstain from VoIP transmission and results in outstanding SU packets in buffers. It is observed from Figure 9 (b) that wasted time intervals increase with decrease in sensing interval. Thus it demands minimum limit on sensing interval. It is also seen from Figure 10 that effective transmission time of SU, free from any PU interference, is high for low sensing intervals. However, this duration is not directly proportional to secondary transmission time interval as increase in effective transmission time is less due to increased PU interference.

\subsection{Advantages of the Designed Model}

Model design is modular in nature as modularity increases simulation speed and allows debugging of errors. Moreover, such a design is encouraged as a valid and simpler model is efficient [26]. In absence of standard for VoIP implementation over CRN, conceptual modeling [27] is followed in this model. Lack of knowledge hinders design of simulation model. Hence, the model is run iteratively to gather data and use it for modifications.

Advantages of adopting an iterative model are stated as follows. 
- It avoids designers from falling in love with a certain model, which is one of the serious perils of simulation design [28].

- It allows scope of introducing agent based modeling [21] where system gradually evolves from interaction of agents.

- Increase in simulation runs with incremental model design detects possible errors and discrepancies in the model [26].

Analysis of simulation data is done graphically as it is simpler and easier to interpret and provides useful information about the trend in variation of parameters. Moreover, lack of standard data for VoIP over CRN proves to be a major obstacle towards proper model design. Therefore, statistical analysis of limited simulation data is not encouraged as such data is easy to misinterpret resulting in gross error [29] and inefficient modeling. Thus this simulation model in Visual C++ serves as an excellent tool to study basic VoIP performance over CRN and provides the researchers with a prototype to implement further optimizations.

\section{VALIDATION}

Validation of the developed models is performed by comparison of simulation data obtained from each model. Figure 11 (a) and Figure 11 (b) show the output obtained from the models in OPNET Modeler 16.0.A and Visual C++ respectively for similar input data. It is observed in both scenarios that increase in sensing period increases SU packet loss which increases as secondary transmission period decreases since packet loss is inversely proportional to the time of VoIP transmission. Similar trends in simulation output validate that designed models provide credible results and can be used for future research studies.

The developed models in OPNET Modeler 16.0.A are built using customizable functions and parameters. While it gives enough flexibility in design and implementation, the process is highly complex and requires repeated compilations, which consume time. Visual $\mathrm{C}++$ is simpler and allows easy customizations but at the cost of less efficient coding. Hence, simpler concepts of centralized architecture are designed in Visual C++ by optimized coding. Distributed architecture requires complex coordination between nodes and hence, OPNET Modeler 16.0.A is selected to implement such principles and develop models accordingly.

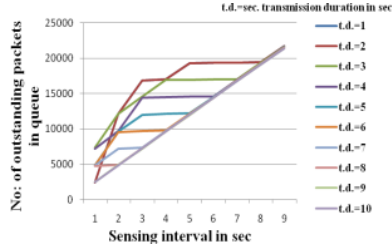

(a)

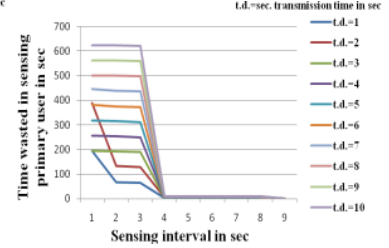

(b)
Figure 9. Variation in (a) number of waiting packets of $\mathrm{SU}$, and (b) sensing time wasted for $\mathrm{PU}$ detection with sensing and transmission intervals

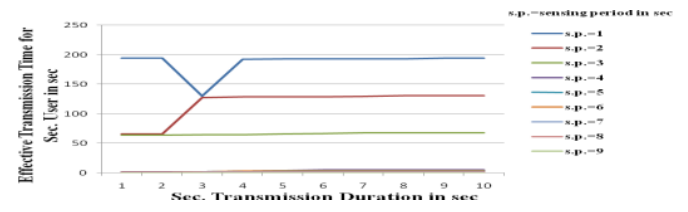

Figure 10. Variation in effective transmission time for $\mathrm{SU}$ with sensing and transmission intervals

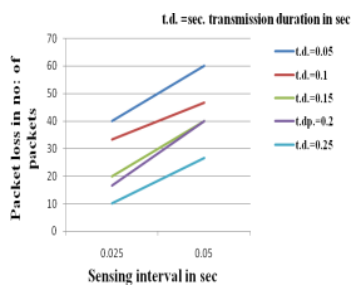

(a)

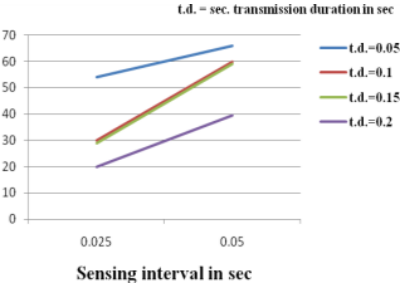

(b)
Figure 11. Variation in packet loss for SU with sensing and transmission intervals in models developed in (a) OPNET Modeler 16.0.A and (b) Visual C++

\section{MATHEMATICAL MODEL}

An exhaustive study of the VoIP model over CRN requires design of a suitable mathematical model to support and validate the obtained output. However, following the principles of prototype modeling, such a mathematical model must be developed initially with basic approximations and gradually that model must be refined to present a more realistic approach. With respect to VoIP over CRN, a detailed mathematical analysis must cover all critical factors ranging from timing parameters (including sensing time, transmission time), PU and SU activities, attributes pertaining to CRN network, VoIP codec parameters, queuing attributes, etc. However, such an analysis is implementation specific and is also dependent on prevailing network conditions and user activity patterns. As this paper primarily aims to provide the initial platform for model development and subsequent research in the domain of VoIP over CRN, a basic mathematical model based on the total time of VoIP transmission is presented that forms the basis of the developed model for VoIP in CRN

The primary analysis is based on the consideration that sensing and transmission time durations are uniform throughout the VoIP communication duration. Moreover, spectral handoff is not considered in this work as it depends on various implementation strategies and spectral handoff policies (proactive and reactive) [30]. Let $T$ be the total time of VoIP transmission that includes the sensing time $t_{s}$ and secondary transmission time $t_{d}$. Let $P_{s i}$ and $P_{d i}$ denote probability of PU arrival in $i$ th sensing period and $i$ th transmission period respectively. The expression for $T$ is derived as follows.

$T=\left[t_{s 1}+\left(1-P_{s 1}\right) t_{d 1}\right]+\left(1-P_{d 1}\right)\left[t_{s 2}+\left(1-P_{s 2}\right) t_{d 2}\right]$
$+\left(1-P_{d 2}\right)\left[t_{s 3}+\left(1-P_{s 3}\right) t_{d 3}\right]+\ldots+\left(1-P_{d n-1}\right)\left[t_{s n}+\left(1-P_{s n}\right) t_{d n}\right]$

Simplifying (2), the expression for $T$ is as follows.

$$
\begin{aligned}
& T=\sum_{i=1}^{n} t_{s i}-\sum_{i=1}^{n-1} P_{d i} t_{s i+1}+\sum_{i=1}^{n}\left(1-P_{s i}\right) t_{d i}(3) \\
& +\sum_{i=1}^{n-1} P_{d i}\left(1-P_{s(i+1)}\right) t_{d(i+1)}
\end{aligned}
$$

Considering fixed $t_{s i}$ and $t_{d i}$ time durations $\forall i ' s$ and simplifying (3),

$$
\begin{aligned}
& T=t_{s}\left[n-(n-1) \sum_{i=1}^{n-1} P_{d i}\right] \\
& +t_{d}\left[n \sum_{i=1}^{n}\left(1-P_{s i}\right)+(n-1) \sum_{i=1}^{n-1} P_{d i}\left(1-P_{s(i+1)}\right)\right]
\end{aligned}
$$

It is apparent that PU arrival probabilities, namely $P_{s i}$ and $P_{d i}$ are interdependent as described below. Let $\lambda$ be the PU arrival probability which is considered to be uniform for every time 
slot in this paper. It is to be noted that when the model is refined for advanced research and learning, $\lambda$ will be governed by the probability distribution function of the corresponding PU traffic distribution.

$$
\begin{aligned}
& P_{d 1}=\lambda\left(1-P_{s 1}\right) \\
& P_{s 2}=\lambda\left(1-P_{d 1}\right)=\lambda\left\{1-\lambda\left(\left(1-P_{s 1}\right)\right)\right\} \\
& T=t_{s}\left[n-(n-1) \sum_{i=1}^{n-1}\left\{\lambda \frac{\left[1+\lambda^{2 i-1}\right]}{1-\lambda^{2}}-\lambda^{2 i-1} P_{s 1}\right\}\right] \\
& +t_{d}\left[n \sum_{i=1}^{n}\left\{1-\left\{\lambda(1+\lambda) \frac{\left[1-\lambda^{2 i-2}\right]}{1-\lambda^{2}}+\lambda^{2(i-1)} P_{s 1}\right\}\right\}\right. \\
& \text { Therefore, } P_{s i} \text { and } P_{d i} \text { for the ith time slot can be } \\
& \text { expressed as follows. } \\
& P_{s i}=\lambda(1+\lambda) \frac{\left[1-\lambda^{2 i-2}\right]}{1-\lambda^{2}}+\lambda^{2(i-1)} P_{s 1} \\
& P_{d i}=\lambda \frac{\left[1+\lambda^{2 i-1}\right]}{1-\lambda^{2}}-\lambda^{2 i-1} P_{s 1}
\end{aligned}
$$$$
+t_{d}\left[n \sum_{i=1}^{n}\left\{1-\left\{\lambda(1+\lambda) \frac{\left[1-\lambda^{2 i-2}\right]}{1-\lambda^{2}}+\lambda^{2(i-1)} P_{s 1}\right\}\right\}+(n-1) \sum_{i=1}^{n-1}\left[\left\{\lambda \frac{\left[1+\lambda^{2 i-1}\right]}{1-\lambda^{2}}-\lambda^{2 i-1} P_{s 1}\right\}\left\{1-\left\{\lambda(1+\lambda) \frac{\left[1-\lambda^{2 i}\right]}{1-\lambda^{2}}+\lambda^{2 i} P_{s 1}\right\}\right\}\right]\right]
$$

$$
\begin{aligned}
& P_{d 2}=\lambda\left(1-P_{s 2}\right)=\lambda\left[1-\lambda\left\{1-\lambda\left(1-P_{s 1}\right)\right\}\right] \\
& \vdots \\
& P_{s n}=\lambda\left(1-P_{d(n-1)}\right)=\lambda\left[1-\lambda\left\{1-\lambda\left\{\ldots . . \lambda\left(1-P_{s 1}\right)\right\}\right\}\right] \\
& P_{d n}=\lambda\left(1-P_{s n}\right)=\lambda\left[1-\lambda\left\{1-\lambda\left\{\ldots . \lambda\left(1-P_{s 1}\right)\right\}\right\}\right]
\end{aligned}
$$

Therefore, $P_{s i}$ and $P_{d i}$ for the $i$ th time slot can be derived and

\subsection{PU Activity}

Thus, the general expression for $T$ can be derived from (4) and expressed in (6). It is clearly observed from the equation that the overall time of VoIP transmission in the most basic scenario depends on the PU traffic characteristics that govern the PU arrival probability $\lambda$. Furthermore, selection of optimal sensing and transmission durations also plays an important role towards deciding the successful VoIP communication duration as inferred from (6).

\section{ANALYSIS OF CRITICAL ATTRIBUTES IN DESIGNED MODELS}

In this section, the critical factors affecting the basic model design for VoIP in CRN are discussed thoroughly. Analysis is carried out based on the obtained simulation data from the OPNET Modeler 16.0.A based Model. OPNET based model is preferred over the Visual $\mathrm{C}++$ based model for analysis in this section as the OPNET based model is more robust and includes the complete set of basic attributes pertaining to VoIP and CRN domain

\subsection{SU Traffic Distribution Pattern}

The work in this paper considers deploying VoIP applications in SUs. Hence, the traffic distribution for SUs is a major factor towards model design and development. It is worth mentioning that VoIP transmission occurs in talkspurts with multiple on-off periods [31]. Therefore, the silence periods in VoIP transmission render the channel idle and also reduce the probability of sudden interference with PU. This fact is clearly established in Figure 12 which shows the variation in channel throughput for varying silence (on and off) periods. Moreover, the variation in SU transmission durations further affects the VoIP talkspurts and the throughput varies accordingly as reflected from Figure 13 (a). However, there is a clear trade-off between increasing SU transmission duration and corresponding decrease in VoIP call quality due to increase in probability of PU interference. Finally, SU traffic distribution is varied in Figure 13 (b) that results in different levels of throughput obtained in case of VoIP SUs for identical transmission durations and on-off periods. Therefore, it can be inferred that accurate estimation of SU traffic pattern is crucial towards achieving high VoIP throughput while maintaining calls at an acceptable quality.
SU activity is further influenced by sudden variations in PU traffic distributions. PUs being the licensed users must be protected from any possible interference with SUs [3]. Therefore, detailed analysis of PU traffic distribution on VoIP throughput is carried out in the developed OPNET based model. It is observed from Figure 14 that increased PU presence results in decrease in SU throughput on the current channel along with increase in SU transmission on the alternative channel. This fact reflects the necessity of carefully selecting alternative channels for $\mathrm{SU}$ transmission in conjunction with channel reservation policies [32] Modifications in sensing and transmission durations along with variation in PU transmission rate further affects interference of SUs with PUs as observed in Figure 15. Finally, the PU traffic distribution pattern is varied to analyze the effect on SU throughput. Figure 16 clearly points out that SU load balancing on multiple channels is a critical aspect that must be studied extensively for different PU transmission distribution functions.

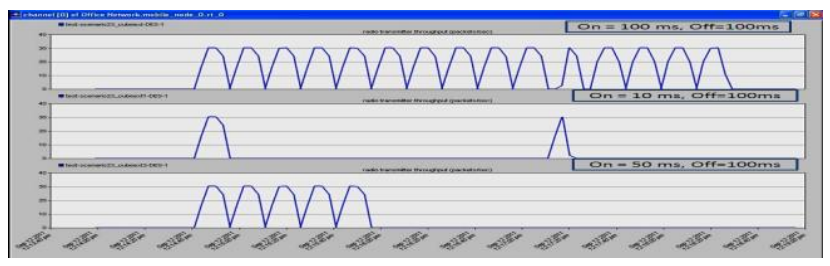

Figure 12. Variation in SU throughput for different on-off periods

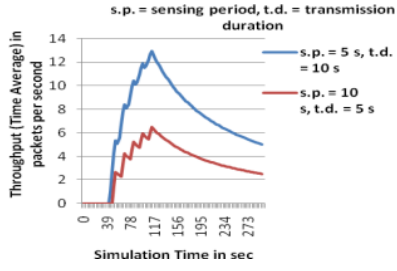

(a)

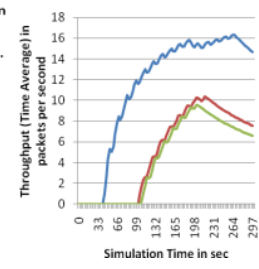

(b)
Figure 13. Variation in SU talkspurt based throughput for (a) different sensing and transmission durations (b) different traffic distributions

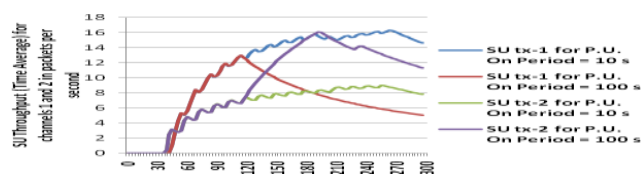

Figure 14. Variation in SU talkspurt based throughput for different PU traffic activity 


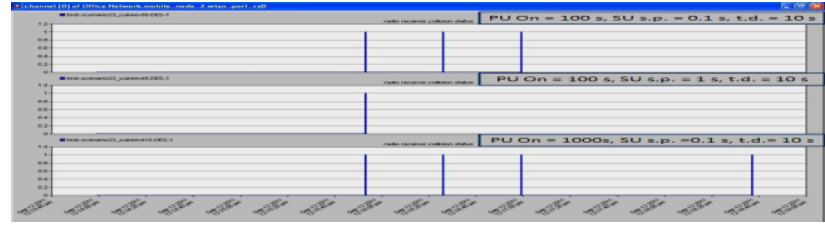

Figure 15. Interference between transmissions of SU and PU for varying PU activity and SU parameters

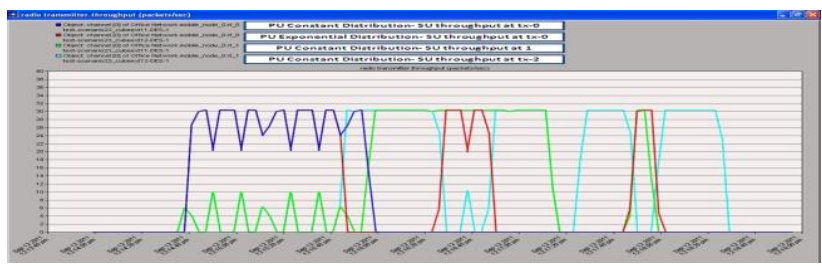

Figure 16. SU throughput in both channels for varying $P U$ traffic distributions

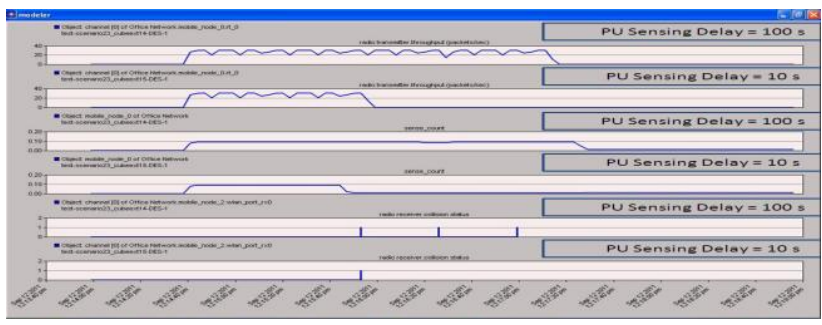

Figure 17. Variation in SU transmission duration, time for which channel is marked as "idle" and collision status with PU for varying delays in sensing PU presence

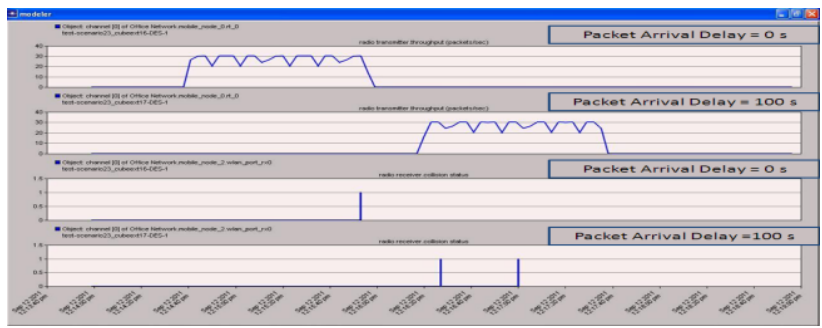

Figure 18. Variation in SU Transmission Time and corresponding $\mathrm{SU}$ interference with $\mathrm{PU}$ for varying packet arrival delays

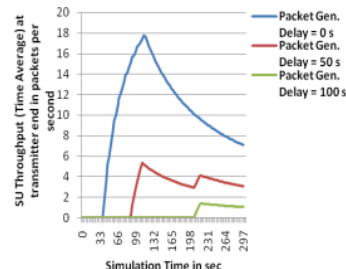

(a)

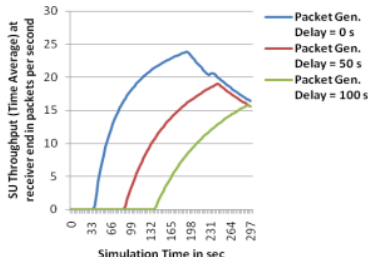

(b)
Figure 19. Variation in SU throughput for different packet generation delays (a) at transmitter end (b) at receiver end

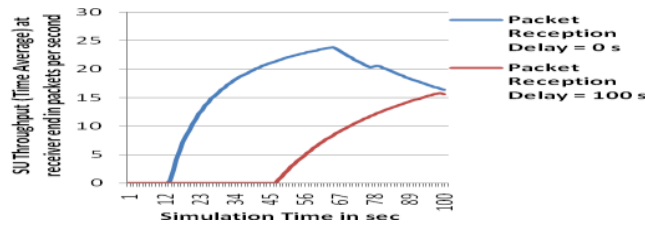

Figure 20. Variation in SU throughput at receiver end for different packet reception delays

\subsection{PU Sensing Issues}

Detailed analysis of the effect of PU presence on SU throughput has already been performed in (i) and (ii). Hence, it is obvious that appropriate PU detection technique must be deployed by SU to avoid any interference and subsequent degradation in VoIP call quality. The reason for applying periodogram technique in this context has been mentioned in Section 3.1.1. However, delays involved in detection of PU and subsequent decision-making can considerably decrease the efficiency of periodogram resulting in interference [17]. This fact is established in Figure 17 where higher delays in sensing PU result in increased duration for which the active channel is marked as idle, creating a scenario of mis-detection [17]. Subsequently, SU transmission time increases, thereby increasing the collision with PUs. Therefore, such delays are highly critical towards a successful VoIP over CRN model and must be reduced.

\subsection{Packet Processing Delays}

Any packet-switched network is characterized by delays involving packet generation, packet processing and reception [33]. Such delays adversely affect the overall quality of transmission and can prove to be critical for real-time VoIP applications in intelligent networks like CRN. This model captures such delays and indicates the corresponding degradation suffered by VoIP applications. It is observed from Figure 18 that the effect of delay in arrival of VoIP packets for transmission results in increased collision with PU. This is because SU transmission does not occur on its designated time slot. Packet generation delay further induces loss in throughput at the transmitter and receiver ends as observed in Figure 19 (a) and Figure 19 (b) respectively. Similarly, the effect of packet reception delay is witnessed in Figure 20 which reflects considerable decrease in throughput at the receiver end. Thus every packet induced delay must be minimized to retain the overall QoS of VoIP in CRN.

\section{CONCLUSION}

This paper has dealt with the design of VoIP model in CRN under various simulation platforms followed by extensive analysis of simulation output. The problem domain is defined along with the basic principle of VoIP implementation in CRN. Initially, VoIP models in CRN domain for single and multi-channel scenarios are designed in OPNET Modeler 16.0.A. following distributed architecture. In the next phase, the principle of centralized architecture is applied to develop a model for VoIP communication over CRN in Visual C++. Detailed analysis of simulation output in each scenario indicates strong correlation between VoIP QoS metrics and CRN parameters. Comparison of the corresponding simulation output validates the proper design of the models. Furthermore, the basic mathematical policy behind the design has been established in this paper that has opened up various issues in both VoIP and CRN domains to be analyzed for future research. Finally, the critical attributes of the developed models are studied in detail to focus on overall quality improvement for VoIP transmission in CRN. Performance 
study of the models reflects efficient simulation design with sufficient credibility. Thus these models can be used by learners and researchers to gather basic knowledge of VoIP transmission in CRN followed by suitable optimizations that are enabled by enormous scope in further development of the designed models.

\section{ACKNOWLEDGMENTS}

The first author deeply acknowledges the support of INSPIRE Fellowship in 2012 from DST, Govt. of India.

\section{REFERENCES}

[1] Federal Communications Commission. Notice of proposed rule making and order: Facilitating opportunities for flexible, efficient, and reliable spectrum use employing cognitive radio technologies. ET Docket No. 03-108.2005.

[2] Y'ucek, T. and Arslan, H. First Quarter 2009. A survey of spectrum sensing algorithms for cognitive radio applications. In Proceedings of IEEE Communications Surveys and Tutorials. vol.11. no.1. pp. 116-130. doi= 10.1109/SURV.2009.090109.

[3] Mitola III, J. and Maguire, Jr G. Q. Aug. 1999. Cognitive radio: making software radios more personal. In Proceedings of IEEE Personal Communications. vol. 6. no. 4. pp. $13-18$. doi $=10.1109 / 98.788210$.

[4] Khasnabish, B. 2003. Implementing Voice over IP. Wiley-Interscience. John Wiley \& Sons, Inc.

[5] Lee, H. and Cho, D. June 2009. VoIP Capacity Analysis in Cognitive Radio System. In Proceedings of IEEE Communication Letters. vol. 13. no. 6. pp. 393-395. doi= 10.1109/LCOMM.2009.082189.

[6] Lee, H. and Cho, D. May 2010. Capacity Improvement and Analysis of VoIP Service in a Cognitive Radio System. IEEE Transactions on Vehicular Technology. vol. 59. no. 4. pp. 1646-1651. doi= 10.1109/TVT.2009.2039503.

[7] Jiang, L., Jiang, T., Wang, Z. and He, X. 23-25, September 2010. VoIP Capacity Analysis in Cognitive Radio System with Single/Multiple Channels. In Proceedings of 6th International Conference on Wireless Communications Networking and Mobile Computing (WiCOM). pp. 1-4. doi= 10.1109/WICOM.2010.5600795.

[8] Zhongliang, L., Shan, F., Dongmei, Z. and Shen, X.S. January 2011. Delay Performance Analysis for Supporting Real-Time Traffic in a Cognitive Radio Sensor Network. IEEE Transactions on Wireless Communications. vol.10. no.1. pp. 325-335. doi= 10.1109/TWC.2010.111910.100804.

[9] Mitola III, J. November 1999. Cognitive radio for flexible mobile multimedia communication. In Proceedings of IEEE International Workshop on Mobile Multimedia Communications (MoMuC). pp. 3-10. doi= 10.1109/MOMUC.1999.819467.

[10] Li, J., Xu, B., Xu, Z., Li, S., and Liu, Y. 2006. Adaptive packet scheduling algorithm for cognitive radio system. In Proceedings of ICCT. pp. 1-5.

[11] Pawlikowski, K., Jeong, H.-D.J., Lee, J.-S.R. Jan 2002. On credibility of simulation studies of telecommunication networks. In Proceedings of IEEE
Communications Magazine. vol.40. no.1. pp.132-139. doi $=10.1109 / 35.978060$.

[12] Sargent, R.J. 5-8 Dec. 2010. Advanced tutorial: Overview of simulation world views. In Proceedings of the 37th Conference on Winter Simulation (WSC). pp. 210-215. doi $=10.1109 /$ WSC.2010.5679161

[13] Hevner, A.R., March, S.T., Park, J and Ram, S. 2004. Design research in information systems research.MIS Quarterly, vol. 28. Issue 1. pp. 75-105.

[14] Rao, D.M. and Wilsey, P.A. 2001. Modeling and simulation of active networks. In 34th Annual Proceedings of Simulation Symposium. pp. 177-184. doi= 10.1109/SIMSYM.2001.922130.

[15] ITU-T. 1972. G.711: Pulse Code Modulation (PCM) of Voice Frequencies.

[16] O. Inc, “OPNET Modeler." [Online]. Available: http://www.opnet.com.

[17] Akyildiz, I. F., Lee, W. Y., Vuran, M. C., and Mohanty, S. Sept. 2006. NeXt generation/dynamic spectrum access / cognitive radio wireless networks: A survey. Computer Networks Journal (Elsevier) 50. pp. 2127- 2159.

[18] Wang, P., Xiao, L., Zhou, S., and Wang, J. 11-15 March 2007. Optimization of detection time for channel efficiency in cognitive radio systems. In Proceedings of IEEE Wireless Communication and Networking Conference. pp. 111-115. Hong Kong. doi= 10.1109/WCNC.2007.26.

[19] Cabric, D., Mishra, S.M., and Brodersen, R.W.7-10 Nov. 2004. Implementation issues in spectrum sensing for cognitive radios. In Conference Record of the ThirtyEighth Asilomar Conference on Signals, Systems and Computers. pp. 772- 776. vol. 1. doi= 10.1109/ACSSC.2004.1399240

[20] Mall, R. 2011. Fundamentals of Software Engineering. PHI Learning Private Limited. $3^{\text {rd }}$ ed.

[21] Pegden, C.D. 5-8 Dec. 2010. Advanced tutorial: Overview of simulation world views. In Proceedings of the 2010 Winter Simulation Conference (WSC). pp. 210215. doi= 10.1109/WSC.2010.5679161.

[22] Soliman, H.M., Elmaghraby, A.S. and El-Sharkawy, M.A. 27-29 July 1995. Parallel and distributed simulation: an overview. In Proceedings of IEEE Symposium on Computers and Communications. pp. 270-276. doi= 10.1109/SCAC.1995.523677.

[23] Balci, O., Nance, R.E., Derrick, E.J., Page, E.H. and Bishop, J.L. 9-12 Dec 1990. Model generation issues in a simulation support environment. In Proceedings of Winter Simulation Conference. pp. 257-263. doi= 10.1109/WSC.1990.129524.

[24] Visual C++ Programmer's Guide. Available: http://msdn.microsoft.com/enus/library/.

[25] Shinn, T. 2006. When is Simulation a ResearchTechnology? Practices, Markets and Lingua Franca. In Proceedings of Simulation, Sociology of the Sciences Yearbook. vol. 25. part 4. pp. 187-203. doi=10.1007/14020-5375-4_12.

[26] Bratley P. Fox B.L. and Schrage L.E. 1987. A guide to simulation, 2nd ed. Springer. 
[27] Robinson, S. 11-14 Dec. 2011. Choosing the right model: Conceptual modeling for simulation. In Proceedings of the 2011 Winter Simulation Conference (WSC). pp.1423-1435. doi= 10.1109/WSC.2011.6147862.

[28] Fritzson, P. 2004. Principles of Object-Oriented Modeling and Simulation with Modelica 2.1. WileyIEEE Press.

[29] Merkuryeva, G. and Vecherinska, O. 24-26 March 2010. Simulation-Based Comparison: An Overview and Case Study. In Proceedings of 12th International Conference on Computer Modelling and Simulation (UKSim). pp. 186-190.

[30] Wang, L., Wang, C. and Chang, C. Sept. 2012. Modeling and Analysis for Spectrum Handoffs in Cognitive Radio Networks. IEEE Transactions on Mobile Computing. vol. 11. no. 9. pp. 1499-1513. doi= 10.1109/TMC.2011.155.
[31] Qaimkhani, I.A. and Hossain, E. January 2008. Efficient silence suppression and call admission control through contention-free medium access for VoIP in WiFi networks. IEEE Communications Magazine. vol.46. no.1. pp. 90-99. doi=10.1109/MCOM.2008.4427236.

[32] Lai, L.J., Ren, L.P., Dutkiewicz, E. and Vesilo, R. 15-18 May 2011. Optimal Channel Reservation in Cooperative Cognitive Radio Networks. In Proceedings of 73rd IEEE Vehicular Technology Conference (VTC Spring). pp. 16. doi=10.1109/VETECS.2011.5956171.

[33] Forgie, J.W. 19-22 May, 1975. Speech transmission in packet-switched store and-forward networks. In Proceedings of AFIPS '75. pp. 137-142. doi= 10.1145/1499949.1499978. 